\title{
Lysophosphatidic acid synthesis and release
}

Céline Pagès, Marie-Françoise Simon, Philippe Valet, Jean Sébastien SaulnierBlache*.

I.N.S.E.R.M U317, Institut Louis Bugnard, Université Paul Sabatier, CHU Rangueil, Batiment L3, 31403, Toulouse cedex 04, France.saulnier@rangueil.inserm.fr

* Corresponding author

Phone: (33)0562172956

Fax: (33)0561331721

Email: saulnier@rangueil.inserm.fr 


\section{Introduction}

First evidence that lysophosphatidic acid (LPA) is not only a key intermediate in glycerolipid synthesis but also a bioactive phospholipid, was brought by Vogt in 1969 (1). This author demonstrated that LPA exerted a constricting activity on isolated rabbit duodenum preparations. From that time, LPA was demonstrated to control numerous cellular functions (smooth muscle contraction, platelet aggregation, $\mathrm{Ca}++$ mobilization , chemotaxis, neurotransmitter release, cell proliferation, cell transformation) via its interaction with specific Gprotein coupled receptors belonging to the Endothelium Differentiation Gene family (EDG2, EDG4 and EDG7) $(2,3)$. Therefore, critical pharmacological concentration of LPA has to be produced extracellularly to induce various receptor-dependent biological responses. The enzymes and proteins involved in synthesis, degradation and transport of LPA control its biodisponibility. Whereas, multiple pathways of synthesis and degradation of LPA have been described, their relative contribution in the extracellular biodisponibility of LPA remains still a matter of debate. Whereas platelets is considered to be the main source of serum LPA, the phospholipid is also found in plasma in physiopathological situations as well as in non-plasmatic extracellular fluids, suggesting the involvement of other cell types in its production.

\section{1- LPA metabolism and transport}

Lysophosphatidic acid (LPA) is the simplest phospholipid and consists of a glycerol backbone with an hydroxyl group at the sn-2 (or sn-1) position, a phosphate group at the sn-3 position, and a fatty acid chain at the sn-1 (or sn-2) position. Most of LPA fatty acids are long chain saturated $(\mathrm{C} 18, \mathrm{C} 16)$ or unsaturated $(18: 1,20: 4)$ fatty acids linked to the glycerol backbone by acyl- or alkyl-group. As shown in figure 1, five different pathways may potentially lead to LPA.

\section{LPA as a precursor of glycerolipid synthesis}


LPA is a key intermediate in early steps of neutral lipid and phospholipid synthesis. The glycerophosphate acyltransferase (GPAT), located in both endoplasmic reticulum and mitochondria catalyses the formation of LPA by acylation of glycerol 3-phosphate. LPA is then rapidly acylated in mitochondria by a monoacylglycerolphosphate acyltransferase (MGAT) in phosphatidic acid (PA), the precursor of all glycerolipids (4). The reduction of acyl dihydroxyacetone phosphate (acyl DHAP) in peroxysomes is also an alternative pathway to LPA production. This latter pathway contributes to LPA formation by pancreatic islets exposed to high glucose concentrations (5). LPA may also be synthetized by the action of a monoacylglycerol kinase (MAG-kinase) on monoacylglycerol, as an important precursor of phosphatidylinositol synthesis (6). MAG-kinase has also been proposed to be involved in the formation of arachidonoyl-LPA in platelets (7). An important question not yet resolved is to determine whether LPA produced during glycerolipid synthesis can acumulate an contribute to the extracellular release of phospholipid.

\section{Synthesis of LPA from PA}

LPA may be synthetized by phospholipase A-catalysed deacylation of phosphatidic acid (PA). Since fatty acids composing LPA can be linked at the sn-1 position (saturated) or at the sn2 position (unsaturated), both PLA2 and PLA1 could be involved in LPA production. Most of LPA species being composed of saturated fatty acids, they likely derive from a PLA2 activity.

Most cells coexpress several types of PLA2: small molecular weight secreted PLA2 (sPLA2), the Ca++-dependent intracellular PLA2 (cPLA2), and the Ca++-independent intracellular PLA2 (iPLA2) (8). The relative contribution of each PLA2 subtype in LPA production remains controversial. Blockers of phospholipase A2 (PLA2) activity such as quinacrine (9) or U10029A (7) inhibit the production of LPA by platelets. Those blockers being poorly selective, their effect do not really inform us about the subtype of PLA2 involved. The proinflammatory type II sPLA2, acts extracellularly and exhibits a high specificity for PA (10). However, considering that most of PA is located in the inner plasma membrane-leaflet (11), a 
rearangement of membrane phospholipid polarity is expected to allow accessibility of PA to sPLA2. Recombinant type II sPLA2 is able to generate LPA in vitro from erythrocyte microvesicules exhibiting an inversion in membrane phospholipid polarity (12). However, thrombin- or phospholipase C-induced LPA production is not altered in platelets isolated from typeII sPLA2 null mice (13). Consequently, type II sPLA2 alone does not contribute or is not sufficient for LPA production by intact cells suggesting the possible involvement of other secreted or intracellular PLA2. In ovarian cancer cell line HEY, LPA production is increased by the protein kinase $\mathrm{C}$ activator PMA, and blocked by the cPLA2/iPLA2 inhibitor AACOCF3 (14). The potential involvement of the iPLA2 in LPA production is particularly interesting since this enzyme exhibits a high substrate selectivity for PA (15). Although less abundant than saturated fatty acid-containing LPA, unsaturated fatty acid-containing LPA(particularly 20:4-LPA) likely positionned in sn-2, also exist. This suggests the possible involvement of a PLA1 in the production of some LPA species. This hypothesis is supported by the identification of a PApreferring PLA1 $(16,17)$. Although LPA can be produced intracellularly by the action of PLA2 or PLA1, it is not yet clear whether this contributes to the extracellular release of the phospholipid.

\section{Synthesis of LPA from lysophosphatidylcholine}

Direct exposition of fibroblast to Streptomyces chromofuscus phospholipase D (PLD) leads to a rapid generation of bioactive LPA associated with a reduction of lysphosphatidylcholine (LPC) (18). Long-term incubation of rat plasma (19) or human follicular fluid (20) promotes the generation of LPA from LPC, suggesting the existence of a circulating lysoPLD. Therefore LPC, or another lysophospholipid, located in the outer plasma membrane leaflet or present in biological fluids constitute potential sources of extracellular LPA. It is noticeable that LPC is found in abundance in oxydized LDL and in culture media conditioned with adipocytes, two important sources of LPA production $(21,22)$.

\section{LPA metabolization}


LPA may rapidly be converted into PA by a LPA-acyltransferase (LPAAT). This enzyme, found in microsomes and plasma membranes is crucial for de novo synthesis of glycerolipids as well as for remodeling of plasma membrane phospholipids (23). The activity of LPAAT being under the control of cAMP-dependent protein kinase (24), the existence of an hormonal control of LPA metabolization can be proposed.

LPA may rapidly be converted into monoacylglycerol (25) by phosphatidate phosphohydrolase (PAP). Although several PAP subtypes exist (26), the existence of an ectoLPA phosphatase, the lipid phosphate phosphohydrolase-1 (LPP1), able to hydrolyse extracellular LPA and to attenuate its biological activity by reducing receptor activation (27) must be noticed.

Finaly, LPA may be hydrolysed and converted into glycerol-3-phosphate by lysophospholipases. Although several lysophospholipases exist (28) a LPA-specific lysophospholipase has been purified from rat brain (29) and could also play an important function by controling LPA availability.

\section{LPA transport}

As mentioned above, LPA can potentially be produced inside and outside the cells. As it is the case for other lipids, intra- and extracellular lipid binding proteins are necessary to avoid LPA toxicity as well as to allow its transportation in plasma and from one cellular organelle to another. Albumin constitutes the main extracellular LPA binding protein with a nanomolar range affinity for the phospholipid and a stoechiometry of about $3 \mathrm{~mol}$ of LPA/mol of albumin (30). Albumin is necessary for the biological activity of LPA, and albumin-bound LPA is responsible for most of heat-stable and lysophospholipase-sensitive biological activities of serum $(31,32)$.

Intracellularly, LPA binds to fatty acid binding proteins (FABP) also known to bind fatty acids. The liver-type FABP (30) exhibits micromolar range affinity for LPA and allows the transport of mitochondrial LPA to microsomes in order to be acylated into PA (33). The potential involvement of FABPs in the transport of LPA to plasma membrane has not yet been tested. 
A third protein which could play an important role in LPA binding and transport is gelsolin. Intracellularly, this protein binds and caps actin filaments. Phosphoinositides of the D3 and D4 types and LPA bind to gelsolin which thus uncaps from the filaments, and provides sites for actin assembly (34). Gelsolin can also be found as a circulating form constituting a potential circulating carrier for LPA (35).

\section{2/ Physiopathological productions of LPA}

\section{LPA and platelet agreggation}

High concentration ( 5 to $25 \mu \mathrm{M}$ ) of LPA is found in serum from different species. Based on qualitative analysis by gaz liquid chromatography the major species of LPA in serum are palmytoyl-, stearoyl, oleoyl- and, to a lesser extend, arachidonoyl-LPA (31, 36-38). Conversely, freshly isolated blood or platelet-poor plasma from healthy individuals contain very low, and often undetectable, concentration of LPA $(31,36,39)$. Aggregating factors such as phospholipase $\mathrm{C}$ or thrombin lead to very significant increase in the production of LPA from isolated platelets (40). The different species of LPA produced by thrombin- activated platelets are similar to those found in serum (7). Therefore, platelets likely contribute to a large part (about 90\%) of serum LPA $(36,41)$ suggesting the existence of other potential sources. LPA has been shown to be generated in vitro by microvesicules sheded from erythrocytes challenged with inflammatory stimuli (12). LPA has also been reported to be produced by myristic acid challenged neutrophiles from aged patients (42). In addition, serum also contains lipoproteins which may also be sources of LPA production (see next section).

\section{LPA, lipoproteins and atherosclerosis}

In human skin fibroblasts the transient increase in intracellular calcium induced by Highdensity lipoproteins (HDL) is desensitized by prior exposure of cells to LPA, suggesting the contribution of the phospholipid in the biological activity of lipoproteins (43). This is supported 
by the presence of relatively high concentration of LPA in the lipid core of pre-beta $1 \mathrm{HDL}$ isolated from human preovulatory follicular fluid (44). LPA is also formed during mild oxidation of low density lipoproteins (LDL) and appears to be an important factor involved in activation of platelets by oxidized LDL. LPA indeed accumulates in human atherosclerotic plaques in vivo, particularly in the lipid-rich core of the lesion (21). It is not yet clear whether lipoprotein LPA results from a phospholipase-catalysed transformation of a precursor (PA, LPC) present in the lipid core, or results from cell-lipoproteins interaction.

\section{Production of LPA in cancer}

An ovarian cancer activating factor identified as LPA was found in plasma and ascites from patients with certain type of ovarian cancer $(45,46)$. LPA concentration can rise up to $20 \mu \mathrm{M}$ in plasma, and is correlated with the degree of cancer malignancy. Plasmatic LPA was therefore proposed to be a potential biomarker of some gynecologic cancers. Conversely to serum LPA, ovarian cancer-LPA is mainly composed of unsaturated fatty acids (45). This suggests that the pathway of production of ovarian cancer-LPA does not involve a PLA2 but rather a PLA1 or a MAG-kinase. Ovarian cancer cells can produce LPA when challenged with a tumor promoting factor (14). Therefore, malignant ovarian epithelial cells may represent an important source of elevated LPA detected in ascites and plasma of patients with ovarian cancers. Nevertheless, metastatic cancers being often associated with local haemorrhage, one cannot exclude that blood cells such as platelets could also be sources for LPA in patients bearing ovarian cancer. Because of its proliferative and transforming activities, LPA produced by cancer cells could represent an important loop of metastasis amplification.

LPA production could also constitute an autocrine loop of amplification of normal cell proliferation. A concomitant production of PA and LPA was indeed detected in Balb/c 3T3 fibroblasts exposed to PDGF (47). Similarly, LPA has also been proposed to be involved in interleukin-1-stimulated mesangial cells (48). 


\section{LPA production and tissue injuries}

Micromolar concentration of LPA has been detected in the aqueous humor bathing injured cornea (49). Although the precise cellular origin of LPA in aqueous humor has not yet been precised, it could play an important role in cornea regeneration by acting on keratinocyte proliferation.

LPA and Endothelin-1 (ET-1) are powerfull activators of cerebrovascular contraction and are released in cerebrospinal fluid (CSF) following cerebral hematoma (50). Intrathecal injection of ET-1 lead to a four fold increase of LPA concentration in CSF (51). Eventhough the precise origin of CSF-LPA remains unclear (cerrebral microvasculature, brain parenchyma or meninges), LPA could be a mediator of ET-1 induced vasoconstriction.

\section{LPA and adipose tissue development}

Adipogenesis corresponds to the recruitment of new adipocytes in adipose tissue and results from proliferation/differentiation of dormant preadipocytes. Production of paracrine and autocrine factors by adipocytes plays an important role in adipogenesis. The existence of an adipocyte production of LPA both in adipocyte conditioned-media and in human adipose tissue dialysate has been demonstrated by our group. This production is increased by catecholamines acting on $\alpha 2$-adrenergic receptors. Adipocyte-LPA present in conditionned-media increases the growth of a preadipose cell line through the activation of Mitogen Activated Protein Kinase (22). Because of the close proximity of preadipocytes and adipocytes within adipose tissue, adipocyte-LPA could play an important role in autocrine/paracrine control of the developement of adipose tissue and connective tissues. Because of the abundance and widespread distribution of the adipose tissue in the organism, particularly in obese individuals, one can speculate that LPA released from adipocytes is an important source of the phospholipid in blood flow.

\section{3- Conclusion}


Several enzymes are potentially involved in LPA synthesis. Some of them act on the lumen side of the cells (sPLA2, lysoPLD) generating direct extracellular production of the phospholipid. Other enzymes act intracellularly (cytosolic PLA2, GPAT, MAGkinase) and their effective contribution in extracellular production of LPA remain still unclear (Figure 1). Although intracellular LPA carriers have been identified, intracellularly produced LPA has to cross plasma membrane. Does LPA translocation requires its metabolization (dephosphorylationphosphorylation; deacylation-acylation) ? Does LPA translocation require already known lipid transporters (translocase, floppase, scramblase, fatty acid-transporters $(52,53))$ ? A great deal of effort will have to be devoted to answer those questions in the future.

Although platelets constitute one of the major source of LPA, several studies have revealed the existence of other cellular (cancer cells, fibroblasts, adipocytes) and non-cellular (lipoproteins) origin of LPA (Figure 2). Therefore, the concept of local (autocrine, paracrine) action of LPA which has been demonstrated in platelet aggregation, has now to be extented to numerous other cellular responses. Moreover, several pathological conditions such as cancer, injuries, hematoma, renal failure, are associated with a relatively high production of LPA, thus constituting an interesting marker of cell agression. Then, LPA being able to control cell proliferation and cell apoptosis, production of LPA by malignant cells could participate in cell survival and tissue regeneration. 


\section{Acknowledgments}

We thank Dr. Max Lafontan for careful reading of the manuscript and helpful comments. 


\section{References}

1. Vogt, W. Pharmacologically active acidic phospholipds and glycolipids. Biochem. Pharmacol. 12:415. 1963.

2. Goetzl, E. and S. An. Diversity of cellular receptors and functions for the lysophospholipid growth factors lysophosphatidic acid and sphingosin 1-phosphate. FASEB J. 12:1589. 1998 .

3. Chun, J., J. J. Contos and D. Munroe. A growing family of receptor genes for lysophosphatidic acid (LPA) and other lysophospholipids (LPs). Cell Biochemistry and Biophysics 30:213. 1999.

4. Haldar, D. and A. Vancura. Glycerophosphate acyltransferase from liver. Methods Enzymol 209:64. 1992.

5. Dunlop, M. E. and R. G. Larkins. Pancreatic islets synthesize phospholipids de novo from glucose via acyl-dihydroxyacetone phosphate. Biochem. Biophys. Res. Commun. 132:467. 1985.

6. Simpson, C., H. Itabe, C. Reynolds, W. King and J. Glomset. Swiss 3 T3 cells preferentially incorporate sn-2-arachidonoyl monoacylglycerol into sn-1-stearoyl-2-arachidonoyl phosphatidylinositol. J Biol Chem 266:15902. 1991.

7. Gerrard, J. M. and P. Robinson. Identification of the molecular species of lysophosphatidic acid produced when platelets are stimulated by thrombin. Biochim. Biophys. Acta. 1001:282. 1989.

8. Dennis, E. Diversity of group types, regulation and function of phospholipase A2. J. Biol. Chem. 269:13057. 1994.

9. McCrea, J., P. Robinson and J. Gerrard. Mepacrine (quinacrine) inhibition of thrombininduced platelet responses can be overcome by lysophosphatidic acid. Biochim Biophys Acta 842:189. 1985.

10. Snitko, Y., E. T. Yoon and W. Cho. High specificity of human secrtory class II phospholipase A2 for phosphatidic acid. Biochem. J. 321:737. 1997. 
11. Zachowski, A. Phospholipids in animal eukaryotic membranes: transverse asymmetry and movement. Biochem J 294:1. 1993.

12. Fourcade, O., M. F. Simon, C. Viodé, N. Rugani, F. Leballe, A. Ragab, B. Fournié, L. Sarda and H. Chap. Secretory phospholipase A2 generates the novel lipid mediator lysophosphatidic acid in membrane microvesicles shed from activated cells. Cell 80:919. 1995.

13. le Balle, F., M. Simon, S. Meijer, O. Fourcade and H. Chap. Membrane sidedness of biosynthetic pathways involved in the production of lysophosphatidic acid. Adv Enzyme Regul 39:275. 1999.

14. Shen, Z., J. Belinson, R. Morton and X. Yan. Phorbol 12-myristate 13-acetate stimulates lysophosphatidic acid secretion from ovarian and cervical cancer cells but not from breast or leukemia cells. Gynecol Oncol 71:364. 1998.

15. Tang, J., R. Kriz, N. Wolfman, M. Shaffer, J. Seehra and S. Jones. A novel cytosolic calcium-independent phospholipase A2 contains eight ankyrin motifs. J. Biol. Chem. 272:8567. 1997.

16. Inoue, M. and H. Okuyama. Phospholipase A1 acting on phosphatidic acid in procine platelet membranes. J. Biol. Chem 259:5083. 1984.

17. Higgs, $H$. and J. Glomset. Identification of a phosphatidic acid-preferring phospholipase A1 from bovine brain and testis. Proc Natl Acad Sci U S A 91:9574. 1994.

18. van Dijk, M., F. Postma, H. Hilkmann, K. Jalink, W. v. Blitterswijk and W. Moolenaar. Exogenous phospholipase D generates lysophosphatidic acid and activates Ras, Rho and Ca2+ signaling pathways. Curr Biol 8:386. 1998.

19. Tokumura, A., K. Harada, K. Fukuzawa and H. Tsukatani. Involvement of lysophospholipase $\mathrm{D}$ in the production of lysophosphatidic acid in rat plasma. Biochim Biophys Acta 875:31. 1986

20. Tokomura, A., M. Miyake, Y. Nishioka, S. Yamano, T. Aono and K. Fukuzawa. Production of lysophosphatidic acids by lysophospholipase D in human follicular fluids of in vitro fertilization patients. Biol Reprod 61:195. 1999. 
21. Siess, W., K. Zangl, M. Essler, M. Bauer, R. Brandl, C. Corrinth, R. Bittman, G. Tigyi

and M. Aepfelbacher. Lysophosphatidic acid mediates the rapid activation of plateletsand endothelial cells by mildly oxidized low densitylipoprotein and accumulates in human atherosclerotic lesions. Proc Natl Acad Sci U S A 96:6931. 1999.

22. Valet, P., C. Pagès, O. Jeanneton, D. Daviaud, P. Barbe, M. Record, J. Saulnier-Blache and M. Lafontan. Alpha2-adrenergic receptor-mediated release of lysophosphatidic acid by adipocytes. J. Clin. Invest. 101:1431. 1998.

23. Bishop, W. and B. RM. Assembly of phospholipids into cellular membranes: biosynthesis, transmembrane movement and intracellular translocation. Annu Rev Cell Biol 4:579. 1988 .

24. Schmidt, A., M. Wolde, C. Thiele, W. Fest, H. Kratzin, A. Podtelejnikov, W. Witke, W. Huttner and H.-D. Soling. Endophilin I mediates synaptic vesicle formation by transfer of arachidonate to lysophosphatidic acid. Nature 401:133. 1999.

25. van der Bend, R., J. d. Widt, E. v. Corven, W. Moolenaar and W. v. Blitterswijk. Metabolic conversion of the biologically active phospholipid, lysophosphatidic acid, in fibroblasts. Biochim Biophys Acta 1125:110. 1992.

26. Brindley, D. and D. Waggoner. Phosphatidate phosphohydrolase and signal transduction. Chem Phys Lipids 80:45. 1996.

27. Jasinska, R., Q.-X. Zhang, C. Pilquil, I. Singh, J. Xu, J. Dewald, D. Dillon, L. Berthiaume, G. Carman, DWWaggoner and D. Brindley. Lipid phosphate phosphohydrolase-1 degrades exogenous glycerolipid and sphingolipid phosphate esters. Biochem J 340:1999.

28. Wang, A. and E. Dennis. Mammalian lysophospholipases. Biochim Biophys Acta 1439:1. 1999.

29. Thompson, F. J. and M. A. Clark. Purification of a lysophosphatidic acid-hydrolysing lysophospholipase from rat brain. Biochem. J. 300:457. 1994.

30. Thumser, A., J. Voysey and D. Wilton. The binding of lysophospholipds to rat liver fatty acid-binding protein and albumin. Biochem J 301:801. 1994. 
31. Tigyi, G. and R. Miledi. Lysophosphatidates bound to serum albumin activate membrane currents in Xenopus oocytes and neurite retraction in PC12 pheochomocytoma cells. J. Biol. Chem. 267:21360. 1992.

32. Ridley, A. J. and A. Hall. The small GTP-Binding protein rho regulates the assembly of focal adhesions and actin stress fibers in response to growth factors. Cell 70:389. 1992.

33. Vancura, A. and D. Haldar. Regulation of mitochondrial and microsomal phospholipid synthesis by liver fatty acid-binding protein. J Biol Chem 267:14353. 1992.

34. Meerschaert, K., V. D. Corte, Y. D. Ville, J. Vandekerckhove and J. Gettemans. Gelsolin and functionally similar actin-binding proteins are regulated by lysophosphatidic acid. EMBO J. 17:5923. 1998.

35. Kwiatkowski, D. Functions of gelsolin: motility, apoptosis, cancer. Curr Opinion Cell Biol 11:103. 1999.

36. Eichholtz, T., K. Jalink, I. Fahrenfort and W. H. Moolenaar. The bioactive phospholipid lysophosphatidic acid is released from activated platelets. Biochem. J. 291:677. 1993.

37. Tokumura, A., M. Iimori, Y. Nishioka, M. Kitahara, M. Sakashita and S. Tanaka. Lysophosphatidic acids induce proliferation of cultured vascular smooth muscles cells from rat aorta. Am. J. Physiol. 267:C204. 1994.

38. Sasagawa, T., K. Suzuki, T. Shiota, T. Kondo and M. Okita. The significance of plasma lysophospholipids in patients with renal failure on hemodialysis. J Nutr Sci Vitaminol 44:809. 1998.

39. Xu, Y., Z. Shen, D. W. Wiper, M. Wu, R. Morton, P. Elson, A. Kennedy, J. Belinson, M. Markman and G. Casey. Lysophosphatidic acid as a potential biomarker for ovarian and other gynecologic cancers. JAMA 280:719. 1998.

40. Mauco, G., H. Chap, M.-F. Simon and L. Douste-Blazy. Phosphatidic and lysophosphatidic acid production in phospholipase $\mathrm{C}$-and thrombin-treated platelets. Possible involvement of a platelet lipase. Biochimie 60:653. 1978. 
41. Gaits, F., O. Fourcade, L. B. F, G. Gueguen, B. Gaigé, A. Gassama-Diagne, J. Fauvel, J.-P. Salles, G. Mauco, M.-F. Simon and H. Chap. Lysophosphatidic acid as a phospholipid mediator: pathways of synthesis. FEBS Lett. 410:54. 1997.

42. Ito, Y., U. Ponnappan and D. Lipschitz. Excess formation of lysophosphatidic acid with age inhibits myristic acid-induced superoxide anion generation in intact human neutrophils. FEBS Lett 394:149. 1996.

43. Porn, M., K. Akerman and J. Slotte. High-density lipoproteins induce a rapid and transient release of $\mathrm{Ca} 2+$ in cultured fibroblasts. Biochem J 279:29. 1991.

44. Jaspard, B., X. Collet, R. Barbaras, J. Manent, C. Vieu, J. Parinaud, H. Chap and B. Perret. Biochemical characterization of pre-beta 1 high-density lipoprotein from human ovarian follicular fluid: evidence for the presence of a lipid core. Biochemistry 35:1352. 1996.

45. Xu, Y., D. Gaudette, J. Boynton, A. Frankel, X. Fang, A. Sharma, J. Hurteau, G. Casey, A. Goodbody, A. Mellors, B. Holub and G. Mills. Characterization of an ovarian cancer activating factor in ascites from ovarian cancer patients. Clin. Cancer Res. 1:1223. 1995.

46. Westermann, A., E. Havik, F. Postma, J. Beijnen, O. Dalesio, M. Moolenar and S. Rodenhuis. Malignant effusions contain lysophosphatidic acid (LPA)-like activity. Ann. Oncol. 9:437. 1998.

47. Fukami, K. and T. Takenawa. Phosphatidic acid that accumulates in platelet-derived groth factor-stimulated Balb/c $3 \mathrm{~T} 3$ cells is a potential mitogenic signal. J. Biol. Chem. 267:10988. 1992.

48. Inoue, C., M. Epstein, H. Forster, O. Hotta, Y. Kondo and K. Iinuma. Lysophosphatidic acid and mesangial cells: implications for renal diseases. Clin Sci (Colch) 96:431. 1999.

49. Liliom, K., Z. Guan, J.-L. Tseng, D. M. Desiderio, G. Tigyi and M. A. Watsky. Growth factor-like phospholipids generated after corneal injury. Am. J. Physiol. 274:C1065. 1998.

50. Tigyi, G., L. Hong, M. Yakubu, H. Parfenova, M. Shibata and C. Leffler. Lysophosphatidic acid alters cerebrovascular reactivity in piglets. Am J Physiol 268:H2048. 1995. 
51. Yakubu, M., K. Liliom, G. Tigyi and C. Leffler. Role of lysophosphatidic acid in endothelin-1- and hematoma-induced alteration of cerebral microcirculation. Am J Physiol 273:R703. 1997.

52. Bevers, E., P. Comfurius, D. Dekkers and R. Zwall. Lipid translocation across the plasma membrane of mammalian cells. Biochem. Biophys. Acta 1439:317. 1999.

53. Hamilton, J. and F. Kamp. How are free fatty acids transported in membranes ? Diabetes 48:2255. 1999. 


\section{Legend of the figures}

\section{Figure 1: LPA metabolism and transport}

Abreviations: LPA (lysophosphatidic acid), PA (phosphatidic acid), MAG (monoacylglycerol), lysoPC (lysophosphatidylcholine), G3P (glycerol-3-phosphate), acylDHAP (acyldihydroxyacetone phosphate), PI (phosphatidyl inositol), PE (phosphatidyl ethanolamine), PC (phosphatidyl choline), PS (phosphatidyl serine), TG (triglyceride), PLA2 (phospholipase A2), LPP1 (lipid phosphate phosphohydrolase-1), PLD (phospholipase D), DAGK (diacyl glycerol kinase), MAGK (monoacyl glycerol kinase) GPAT (glycerophosphate acyl transferase), MGAT (monoacyl glycerolphosphate acyl transferase), FABP (fatty acid binding protein)

\section{Figure 2: Cellular and non-cellular sources of LPA}

